\title{
Design \& Development of Automatic Conveyor Controlling System for Sorting of Component on Color basis
}

\author{
Amir Deshmukh ${ }^{1}$, Mahesh Nagane ${ }^{2}$, Vaibhav Awatade $^{3}$ \\ ${ }^{1}$ Department of Mechanical Engineering, S.M.S.M.P. Institute of Technology \& Research center, Akluj 413101, Maharashtra, India
}

\begin{abstract}
In many packaging industries, color object sorting is the major task that needs to be done at final dispatch section. Sorting of various products in such industries is accomplished based on appearance i.e. color, shape and sizes. Manual sorting is the tradition approach that preferred by industries that involves visual inspection performed by human operators. This traditional approach is tedious, time-consuming, slow and non-consistent. It has become increasingly difficult to hire personnel who are adequately trained and willing to undertake the tedious task of inspection. Therefore the efforts are made to design and implementation of automatic technique to determine color of object, color based component sorting using microcontroller. In implemented system, colored object which is rolling over a conveyer belt has been detected by using color sensors. Using color sensors, the color of a component which may be red, green or blue has been determined. Once the color of a component is determined, implemented system will automatically count and sort the objects as per its color.
\end{abstract}

Keywords: Conveyor system, Color sensors, Color theory, Component sorting, Microcontroller.

\section{Introduction}

We have proposed simple, low cost, low power consumption component sorting unit based on its color. Where it is placed on conveyor belt which is running continuously with the help of D.C. motor. The objective of the project is to sort the component according to color. Our proposed works aims at the testing of the manufactured component by an automated way instead of using manual way. It is aimed to reduce human effort and at the same time increase the productivity \& accuracy levels that cannot be achieved with manual operations.

\section{Problem Identification}

In the speed running world everyone are considering the time factor as an important issues. To reduce this time or managing this time, a small implementation which is useful to industries is our project. Today in industries, same model or same object is manufactured with little variation like weight, size etc. For placing the same type of object from one place to other place, sorting them on color and size basis we use labor. So for this all industries will spend huge amount as wages and take lot of time for processing. By considering this entire project which make all this i.e. Sorting of object based on color, counting the objects which were sorted and display of its counting would be better choice.

\section{Literature Survey}

Most sensors are electrical or electronic, although other types exist. A sensor is type of transducer. Sensors are either direct indicating (e.g. a mercury thermometer or electrical meter) or are paired with an indicator (perhaps indirectly through an analog to digital converter, a computer and a display) so that the value sensed becomes human readable.
In addition to other applications, sensors are heavily used in medicine, industry and robotics. A common requirement in the field of color sensing is that of color identification, or sorting of objects by color. Typically this type of application is simpler than a general-purpose color measurement application. A common task in color sensing is to identify an unknown color as falling into one of these general categories.

\subsection{Color Identification}

Color names can be used and conjure reasonably consistent perceptions. There have eleven basic color names have been identified such as white, gray, black, red, yellow, green, blue, orange, purple, pink, and brown. Most or all colors can be described in terms of variations and combinations of these colors. Due to the fact that human color vision is accomplished in part by three different types of cone cells in the retina, it follows that three values are necessary and sufficient to define any color.

\subsection{Color Theory}

Berlien (2004) was said that there has three values can be thought of as coordinates of a point in three-dimensional space, giving rise to the concept of color space. Hue, saturation, luminance (HSL) is one such color coordinate system, or color space. A more precise method of describing color is by hue, saturation, and lightness. Hue is the attribute of a color according to its similarity with one of the colors red, yellow, green, or blue, or a combination of adjacent pairs of these colors considered in a closed ring, as shown in this figure 3.1 


\section{International Journal of Science and Research (IJSR) \\ ISSN (Online): 2319-7064}

Index Copernicus Value (2013): 6.14 | Impact Factor (2014): 5.611
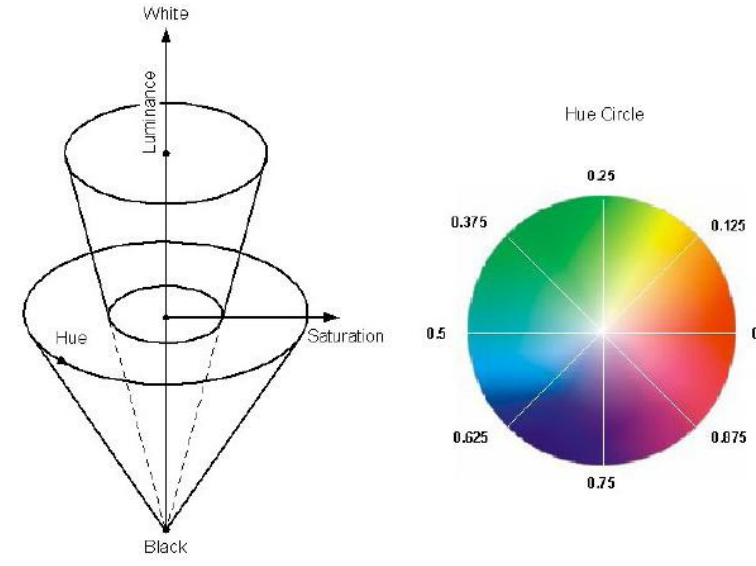

Figure 2.1: HSL diagram with Hue circle (Berlien 2004)

\subsection{Color and Light}

A „color" is an interaction between a very small range of electromagnetic waves and the eyes and brain of a person. What people call red, green, or blue are just ways of categorizing what their brain experiences. An article by Bishop and Lee (2006) brief that, the spectrum of light where the eye can see is called the visible region as can be seen in figure 2.4. Light is a type of energy, which makes up a small portion of the electromagnetic spectrum. Visible light could be expressed as a frequency, but the magnitude is so large people generally express the wavelength of light in units of nanometers (10-9 meters) to describe light. The region of visible light consists of light with a wavelength between approximately $380 \mathrm{~nm}$ to $780 \mathrm{~nm}$. The visible colors and their corresponding range of wavelengths can be found in Table 2.1

Table 2.1: Color vs. Wavelength range

\begin{tabular}{|c|c|}
\hline Color & Wavelength range $(\mathrm{nm})$ \\
\hline violet & $380 \sim 410$ \\
\hline Indigo & $410 \sim 450$ \\
\hline Blue & $450 \sim 510$ \\
\hline Green & $510 \sim 560$ \\
\hline Yellow & $560 \sim 600$ \\
\hline Orange & $600 \sim 630$ \\
\hline Red & $630 \sim 780$ \\
\hline
\end{tabular}

\subsection{Color Sensor}

Color sensors register items by contrast, true color, or translucent index. True color sensors are based on one of the color models, most commonly the RGB model. A large percentage of the visible spectrum can be created using these three primary colors. Many color sensors are able to detect more than one color for multiple color sorting applications. Depending on the sophistication of the sensor, it can be programmed to recognize only one color, or multiple color types or shades for sorting operations. Some types of color sensors do not recognize colors, instead focusing on light wavelengths. Sensor can be configured to locate wavelengths from near infrared (colors in the $750 \mathrm{~nm}$ to $2500 \mathrm{~nm}$ wavelength range), far infrared (colors in the 6.00 to 15.00 micron wavelength range), and UV (colors in the 50 to 350 and $400 \mathrm{~nm}$ wavelength range) in addition to the visible range. Sensors that read the visible range are the most common type of color sensors. They measure color based on an RGB color model. A large percentage of the visible spectrum (380 nm to $750 \mathrm{~nm}$ wavelength) can be created using these three colors. Color sensors are generally used for two specific applications:

\section{True Color Recognition}

Sensors used for true color recognition are required to "see" different colors or to distinguish between shades of a specific color. They can be used in either a sorting or matching mode. In sorting mode, output is activated when the object to be identified is close to the set color. In matching mode, output is activated when the object to be detected is identical (within tolerance) to the color stored in memory.

\section{Color Mark Detection}

Color mark detection sensors do not detect the color of the mark; rather they "see" differences or changes in the mark in contrast with other marks or backgrounds. They are sometimes referred to as contrast sensors. Color sensors shine light onto the object to be monitored and measure either the direct reflection or the output into color components. Many color sensors have integral light sources to achieve the desired effect. These integral light sources include LEDs, lasers, fiber optic, and halogen lamps.

\subsection{Sorting}

Sorting is any process of arranging items in some sequence and/or in different sets. It has two common distinct meanings such as ordering and categorizing. Ordering is arranging items of the same kind, class, nature, etc. in some ordered sequence while categorizing is grouping and labeling items with similar properties together by sorts.

\section{1)Sorting Information or Data}

One important kind of sorting is arranging items of information in alphabetical sequence according to some pre-defined ordering relation (sort key by each group of lists), e.g. when one sorts the books in a library by title, subject or author (all alphabetically sorted normally in ascending order). The resulting order may be either ascending or descending, because essentially all sorting is numerical sorting. The main purpose of sorting information is to optimize its usefulness for specific tasks.

\section{2)Physical Sorting Processes}

Various sorting tasks are essential in industrial processes. For example, during the extraction of gold from ore, a device called a shaker table uses gravity, vibration, and flow to separate gold from lighter materials in the ore (sorting by size and weight). Sorting is also a naturally occurring process that results in the concentration of ore. Sorting results from the application of some criterion or differential stressor to a mass to separate it into its components based on some variable quality. Materials that are different, but only slightly such as the isotopes of uranium, are very difficult to separate.

\subsection{Conveyor belt}

The history of conveyor belts begins in the latter half of the 17 th century. Since then, conveyor belts have been an 


\section{International Journal of Science and Research (IJSR) \\ ISSN (Online): 2319-7064}

Index Copernicus Value (2013): 6.14 | Impact Factor (2014): 5.611

inevitable part of material transportation. But it was in 1795 that conveyor belts became popular means for conveying bulk materials. In the beginning, conveyor belts were used only for moving grain sacks to short distances. Conveyor belt system and working were quite simple in the early days. The conveyor belt system had a flat wooden bed and a belt that traveled over the wooden bed. Earlier, conveyor belts were made of leather, canvas or rubber. This primitive conveyor belt system was very popular for conveying bulky items from one place to another. In the beginning of the 20th century, the applications of conveyor belts became wider.

Hymle Goddard of Logan Company was the first to receive the patent for the roller conveyor in 1908. The roller conveyor business did not prosper. A few years later, in 1919, powered and free conveyors were used in automotive production. Thus, conveyor belts became popular tools for conveying heavy and large goods within factories. During the 1920s, conveyor belts were common, and also underwent tremendous changes. Conveyor belts were used in coal mines to handle runs of coal for more than $8 \mathrm{kms}$, and were made using layers of cotton and rubber covers. The longest conveyor belt now in use is 60 miles long, in the phosphate mines of Western Sahara. One of the turning points in the history of conveyor belts was the introduction of synthetic conveyor belts. It was introduced during the Second World War, mainly because of the scarcity of natural materials such as cotton, rubber and canvas. Since then, synthetic conveyor belts have become popular in various fields. With the increasing demand in the market, many synthetic polymers and fabrics began to be used in the manufacture of conveyor belts. Today, cotton, canvas, EPDM, leather, neoprene, nylon, polyester, polyurethane, urethane, PVC, rubber, silicone and steel are commonly used in conveyor belts. Nowadays, the material used for making a conveyor belt is determined by its application.

\section{System Technology}

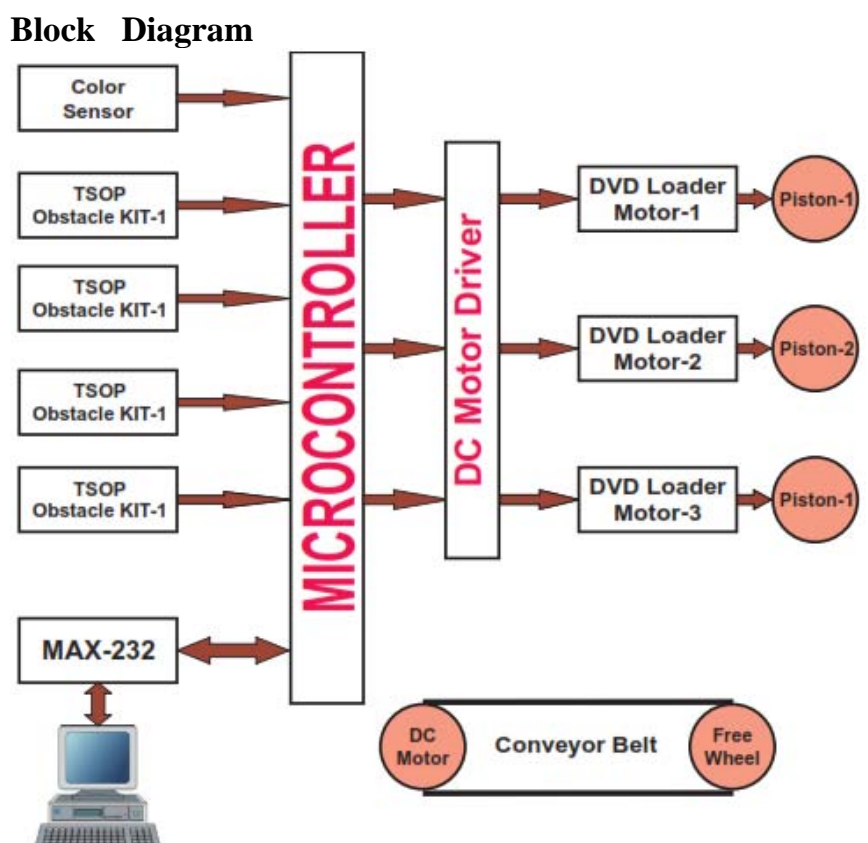

Figure 4.1: System block diagram

\subsection{Color sensor}

This color sensor identifies color and gives serial output of RBG value. It can identify 16.7 million color shades giving RGB value for the detected color. The detected color is identified as amount of three primary color values namely Red, Green \& Blue with 8 bit accuracy for each primary color. Any color can be separated or combined into three primary colors Red, Green and Blue using the RBG values.

\subsection{TSOP 1738}

The TSOP17 series are miniaturized receivers for infrared remote control systems. PIN diode and preamplifier are assembled on lead frame, the epoxy package is designed as IR filter. The demodulated output signal can directly be decoded by a microprocessor. TSOP17 is the standard IR remote control receiver series, supporting all major transmission codes

\subsection{PIC Microcontroller (PIC 16F877A)}

Microchip manufacture a series of microcontrollers called PIC. There are many different flavors ${ }^{e e}$ available, some basic low memory types, going right up through to ones that have Analogue - To- Digital converters and even PWM built in. We are going to concentrate on the 16F877A PIC. Once you have learnt how to program one type of PIC, learning the rest is easy. There are several ways of programming the PIC - using BASIC, C, or Assembly Language. We are going to use the assembly language There are only 35 instructions to learn, and it is the cheapest way to program the PICs, as you do not need any extra software other than the freebies.

\subsection{Supply voltage}

Most microcontrollers operate with the standard logic voltage of _5V. A voltage regulator circuit is usually used to obtain the required power supply voltage when the device is to be operated from a mains adaptor or batteries.

\subsection{The clock}

All microcontrollers require a clock (or an oscillator) to operate. The clock is usually provided by connecting external timing devices to the microcontroller. An instruction is executed by fetching it from the memory and then decoding it. This usually takes several clock cycles and is known as the instruction cycle.

\subsection{Timers}

Timers are important parts of any microcontroller. A timer is basically a counter which is driven either from an external clock pulse or from the internal oscillator of the microcontroller. A timer can be 8-bits or 16-bits wide.

\subsection{Reset input}

A reset input is used to reset a microcontroller. Resetting puts the microcontroller into a known state such that the 


\section{International Journal of Science and Research (IJSR) \\ ISSN (Online): 2319-7064}

Index Copernicus Value (2013): 6.14 | Impact Factor (2014): 5.611

program execution starts from address 0 of the program memory.

\subsection{Interrupts}

An interrupt causes the microcontroller to respond to external and internal (e.g. a timer) events very quickly. When an interrupt occurs the microcontroller leaves its normal flow of program execution and jumps to a special part of the program. Another important feature with multiinterrupt capability is that different interrupt sources can be given different levels of priority.

\subsection{Analogue-to-digital converter}

An analogue-to-digital converter (A/D) is used to convert an analogue signal such as voltage to a digital form so that it can be read by a microcontroller. Some microcontrollers have built-in A/D converters.

\subsection{Serial $\mathrm{I} / 0$}

Serial communication (also called MAX232 communication) enables a microcontroller to be connected to another microcontroller or to a PC using a serial cable. The baud rate and the data format can usually be selected by the user program. If any serial I/O hardware is not provided, it is easy to develop software to implement serial data communication using any $\mathrm{I} / \mathrm{O}$ pin of a microcontroller.

\subsection{EEPROM data memory}

EEPROM type data memory is also very common in many microcontrollers. The advantage of an EEPROM memory is that the programmer can store non-volatile data in such a memory, and can also change this data whenever required. Then, if the power supply is removed for whatever reason, the values of the latest readings will still be available in the EEPROM memory. PicBasic and PicBasic Pro languages provide special instructions for reading and writing to the EEPROM memory of a microcontroller which has such memory built-in.

\subsection{MAX-232-}

Actually MAX 232 is used because we wanted to have serial communication between microcontroller and computer. This supports short distance serial communication. We are using MAX 232 in IC format due to readymade availability of this. Some capacitors have to arrange along peripherals pins of this IC which we have connected in our actual circuit. Need of these capacitors is due to fact that MAX 232 datasheet shows need of such capacitors in order to pull data from one point to another point.

The MAX 232 is a dual driver/receiver that includes a capacitive voltage generator to supply TIA/EIA-232-F voltage levels from a single $5-\mathrm{V}$ supply. Each receiver converts TIA/EIA-232-F inputs to 5-V TTL/CMOS levels. These receivers have a typical threshold of $1.3 \mathrm{~V}$, a typical hysteresis of $0.5 \mathrm{~V}$, and can accept $\pm 30-\mathrm{V}$ inputs. Each driver converts TTL/CMOS input levels into TIA/EIA-232-F levels.

\subsection{Dc motors}

A DC motor usually means a permanent-magnet, directcurrent (DC) motor of the sort used in toys, models, cordless tools, and robots. These motors are particularly versatile because both their speed and direction can be readily controlled; speed by the voltage or duty cycle of their power supply, and direction by its polarity. Torque is a measurement of the motors power. The higher the torque of the motor the more weight it can move. DC motors provide different amounts of torque depending on their running speed, which is measured in RPM (revolutions per minute). At low RPM DC motors produce poor torque, and generally the higher the RPM, the better the motors torque. However, in high torque, the speed may be too high for an application. That's why we have to use gears (or geared motor) to reduce the overall speed of the motor and running at the top speed to get the most power to, say, a wheel attached to the shaft of the motor.

\section{Working}

\section{Circuit Action}

The sensor switches each primary color RGB, one by one and checks what intensity of color is reflected by the surface of detection. This reflected intensity is converted to 8 bit value. For example a RED surface will strongly reflect RED. While a Yellow surface will reflect RED and GREEN both. According to the induction principle of the three primary colors which create various other colors in nature, once the value of three primary colors is confirmed, the color of the tested object is known. Knowing the value of RGB helps people gain the color of the light which is projected onto the sensor since each color correspond to only one value of RGB. For example When RED shade of color is detected you would get following type of data in terminal Red $=130$ Green $=030$ Blue $=030 \quad \mathrm{~L}=010$ This value read the microcontroller and sends the signal to particular stepper motor. Hear the stepper motor is used as piston to push the corresponding object. Before piston we use IR receiver to detect the object and for counting also. The current buffer IC ULN2003A use to drive the stepper motor. The main working - For example RED object is detected then microcontroller waits for cut red piston IR signal then red piston stepper motor rotate and red object push from conveyer belt. And count the RED Object and the data sends to PC. And so on A VB program is design to display the entire object counted value.

\section{Result \& Discussion}

\subsection{Result}

The final result was quite satisfactory. The color detecting sensors worked well and it was able to detect red or green or blue object quite nicely and change the direction of servo on right and left side to sort the object in proper place. Geared DC motor was used to get increased torque for the movement of the shaft as well as the conveyor belt. The belt 


\section{International Journal of Science and Research (IJSR) \\ ISSN (Online): 2319-7064}

Index Copernicus Value (2013): 6.14 | Impact Factor (2014): 5.611

moved from starting point to the end point through the roller without conflicting with the walls. The system performed well as programmed and detects the object according to their color.

\subsection{Limitation}

Every successful invention has its own limitations. There are a number of limitations in the project also, which are as follows:

a) Here in case of color measurement and detection of color over a wide range which might not gave the proper result always.

b) Here only two colored (red and green) products are asserted precisely. Other color can be asserted by preparing a good and more accurate structure and highly efficient coding of microcontroller.

c) Sometime the belt slip on the roller as gripping mechanism of belt with the roller is not good enough. Again no use of timing belt and gear also causes the faulty power transmission to the roller.

\subsection{Further Development}

Anyone can upgrade the system using the following instructions:

a) Using of timing belt and gear instead of direct connection with roller and motor shaft will be more efficient. But care should be taken with the proper meshing of timing belt and gear.

b) Some rubber gripers can be used. It increases surface resistance which helps to avoid slipping of conveyor belt.

c) Using of TCS230 color sensor can be more useful. But it is not cost effective.

d)A pneumatic actuator can be used for sorting and placing the objects in different belt.

\section{Conclusion}

Nowadays in highly competitive industrial manufacturing, the management of the integrity of supply of a product from raw material to finished product through quality manufacturing is of paramount importance. For the declaration of a product bearing high quality and dimensional accuracy is mandatory. So this project of automatic color sorting is an excellent one because of its working principle and wide implementation. By applying the idea of this project an industry can easily sort the required product according to its color. Though it has some limitations, but by having done some modification this concept can be implemented in wide range of application.

\section{Acknowledgement}

We would like to express our gratitude and appreciation to our guide, Mr. Ramdas Bochare, Head of Mechanical Engineering Department, S.M.S.M.P institute of technology and research, Akluj for his positive attitude and guidance which is very helpful for the completion of the project. We would also like to express our sincere gratitude to all of our respective teachers for their support. Finally thanks to almighty for everything he made possible.

\section{References}

[1] Hiwa Golpira, Hêmin Golpîra, "Improvement of an Apple Sorting Machine Using PSNR Criterion," Advanced Mechatronics System Japan, Sept. 2012, pp. 729-732.

[2] Mohammad Moghaddam Vahed, Sattom Halder, Mahmood Sabria Chowdhury and S. C. Banik, "Study of Automatic Sorting System for Date Fruits," International Conference on Mechanical Engineering and Renewable Energy, May 2014, pp. 219-224.

[3] P.B.Vijayalaxmi, Rohan Putta, Gayatri Shinde, Punit Lohani, "Object Detection Using Image Processing for an Industrial Robot," International Journal of Advanced Computational Engineering and Networking, Sept-2013, pp. 21-26.

[4] Smita Shelke, A.P Patale, "An Automatic Grading System based on A Machine Vision," International Journal of Innovative Research in Electrical, Electronics, Instrumentation and Control Engineering, July-2013, pp.154-160.

[5] Vishnu R. Kale, V. A. Kulkarni, "Automation of Object Sorting System Using Pick \& Place Robotic Arm \& Image Processing," Proceedings of 3rd IRAJ International Conference, January 2014, pp. 56-60.

[6] S. Tsalidis, Dentsoras, "Application of Design Parameters Space Search for Belt Conveyor Design" Journal of Plant Science, Vol. 10, No. 6, pp. 617-629, 2010

[7] Huang, Wang, Mei, Zhao, "Time Minimum Trajectory Planning of a 2-DOF Translational Parallel Robot for Pick-and-place Operations" IEEE Computer Magazine, Vol. 56, No. 10, pp. 365-368, 2007. 